\title{
Calculation of the Cherenkov-light yield from electromagnetic cascades in ice with Geant4
}

\author{
Leif Rädel $^{\mathrm{a}}$, Christopher Wiebusch ${ }^{\mathrm{a}}$ \\ ${ }^{a}$ III.Physikalisches Institut, Physikzentrum, RWTH Aachen University, Otto Blumenthalstrasse, \\ 52074 Aachen, Germany
}

\begin{abstract}
In this work we investigate and parameterize the amount and angular distribution of Cherenkov photons which are generated by electromagnetic cascades in water or ice. We simulate electromagnetic cascades with Geant 4 for primary electrons, positrons and photons with energies ranging from $1 \mathrm{GeV}$ to $10 \mathrm{TeV}$. We parameterize the total Cherenkov light yield as a function of energy, the longitudinal evolution of the Cherenkov emission along the cascade-axis and the angular distribution of photons. Furthermore, we investigate the fluctuations of the total light yield, the fluctuations in azimuth and changes of the emission with increasing age of the cascade.
\end{abstract}

Keywords: Neutrino telescopes, Cherenkov-light, Geant4, electro-magnetic cascades

\section{Introduction}

High-energy neutrino telescopes such as IceCube, Baikal or Antares [1, 2, 3] detect Cherenkov-light from charged particles in natural media like water or ice. Cherenkov-light is produced when these particles propagate through the medium with a speed faster than the phase velocity of light $v>c_{\text {med }}=c / n$. In ice and water the refraction index $n$ is typically $n \approx 1.33$ [4, 5]. Hence, the Cherenkov-threshold is given by $\beta=\frac{1}{n}$ which corresponds to a minimum kinetic energy of

$$
E_{c}=m \cdot\left(\frac{1}{\sqrt{1-\frac{1}{n^{2}}}}-1\right)
$$

\footnotetext{
is published in Astroparticle Physics 44 (2013) 102113

Email addresses: Leif.Raedel@physik.rwth-aachen.de (Leif Rädel), Christopher.Wiebusch@physik.rwth-aachen.de (Christopher Wiebusch)
} 
For electrons this is $E_{c} \approx 0.26 \mathrm{MeV}$ in water and ice. The number of emitted photons per unit track and wavelength interval is given by the Frank-Tamm formula [6, 7]

$$
\frac{d^{2} N}{d x d \lambda}=\frac{2 \pi \alpha z^{2}}{\lambda^{2}} \cdot \sin ^{2}\left(\theta_{c}\right) .
$$

Here $\theta_{c}$ is the Cherenkov-angle. This is the opening angle of a cone into which the photons are emitted

$$
\cos \left(\theta_{c}\right)=\frac{1}{n \beta} .
$$

A relativistic track $(\beta=1)$ in water or ice $(n \approx 1.33)$ produces about $N_{0} \approx 250 \mathrm{~cm}^{-1}$ optical photons in a wavelength interval between $300 \mathrm{~nm}$ and $500 \mathrm{~nm}$, which is a typical sensitive region of photo-detectors, e.g. [8], used in the aforementioned neutrino telescopes. The Cherenkov-angle for a relativistic track $(\beta=1)$ in ice is $\theta_{c, 0}=\arccos (1 / n) \approx 41^{\circ}$.

A large fraction of detected Cherenkov-photons in high-energy neutrino telescopes originates from electromagnetic cascades. These are initiated by a highenergy electromagnetic particle which produces a shower of secondary particles by subsequent bremsstrahlung and pair production processes [6, 9]. The primary particle can originate from radiative energy losses of a high-energy muon (bremsstrahlung and pair production), or from the decay of $\pi^{0} \rightarrow 2 \gamma$ in hadronic cascades, or be a high-energy electron from a charged-current interaction of an electron neutrino.

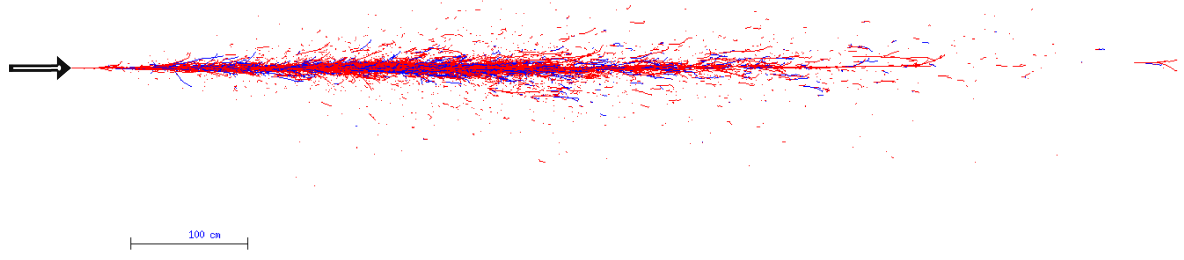

Figure 1: A simulated electromagnetic cascade. A primary electron of $100 \mathrm{GeV}$ has been injected at the left pointing towards the right. Shown are all generated charged secondary particles (red for negative and blue for positive charge) as the result of a Geant 4 simulation. Neutral particles, like photons are not shown. 
An example of a simulated cascade is shown in figure 1. Each particle in the cascade produces Cherenkov-light according to Eqs.(2) and (3), if its energy is above the Cherenkov-threshold Eq.(1). Due to multiple interactions and scattering the directions of the particles in the cascade differ from that of the original primary particle and a broad angular distribution of emitted Cherenkov-photons is expected.

The characteristic length scale for the development of an electromagnetic cascade is given by the radiation length $X_{0}$ [6]. It is about $X_{0, \text { ice }} \approx 39.75 \mathrm{~cm}$ and $X_{0, \text { water }} \approx 36.08 \mathrm{~cm}$ as determined by Geant 4 for the configuration listed in Appendix A. The length along the shower axis $z$ is usually expressed by the dimensionless shower depth

$$
t \equiv z / X_{0}
$$

The length of the shower increases typically logarithmically with the ratio of primary energy $E_{0}$ and critical energy $E_{c r i t}$. The critical energy is the energy above which radiative process dominate the energy loss of electrons. The values for ice and water obtained from [10] are $E_{\text {crit,ice }}^{\mathrm{e}-}=78.60 \mathrm{MeV}, E_{c r i t, i c e}^{\mathrm{e}+}=76.51 \mathrm{MeV}$ and $E_{\text {crit,water }}^{\mathrm{e}-}=78.33 \mathrm{MeV}, E_{\text {water, }, \text { ice }}^{\mathrm{e}+}=76.24 \mathrm{MeV}$.

The physical length of a shower is typically less than $10 \mathrm{~m}$. This is short, compared to the scale of neutrino telescopes and the full Cherenkov-light is created locally and expands with time as an almost spherical shell with a characteristic angular distribution of the intensity.

Due to the large number of particles the full tracking of each particle in MonteCarlo simulations of cascades in neutrino telescopes is very time consuming. However, the development of electromagnetic cascades is very regular because fluctuations are statistically suppressed by the large number of interactions and large number of involved particles. Hence, it can be well approximated by the average development. Therefore, for the simulation of data in neutrino telescopes, the average Cherenkov-light output can be parameterized, e.g. as done in [11, 12, 13, 14].

This work follows up the work in [11] which was based on Geant3.16 [15] with a more precise calculation of the total Cherenkov-light yield and its angular distribution based on Geant4 [16]. For different primary energies and primary particles, we investigate the velocity distribution and the directional distribution of particles and the longitudinal development of the cascade. We present a parameterization of the Cherenkov-light yield and investigate its fluctuations as well as variations of the azimuthal symmetry of the cascade. We also present a parameterization of the angular distribution of Cherenkov-photons and investigate variations of this distribution during the development of the cascade. The results are compared to [11, 12, 13, 14].

We note, that similar calculations have been also been performed for the calcualtion of coherent radio emission from electro-magnetic cascades in ice [17]. 
Although those calculations do not consider the photon yield of Cherenkov light, and concentrate on the radio emission their results e.g. for the total track-length are similar.

\section{Simulation method}

The calculation of this work follows largely the strategy described in [18]. We use the Geant4 (GEometry ANd Tracking) toolkit to track the particles in the cascade through the medium ice or water [16]. The used media properties are given in Appendix A. Unless noted otherwise, we used an index of refraction of $n=1.33$ and a density of $\rho_{\text {ice }}=0.91 \mathrm{~g} / \mathrm{cm}^{3}$. Note, that these values slightly deviate from the values in [10]: $\rho_{\text {ice }}=0.918 \mathrm{~g} / \mathrm{cm}^{3}$ and $n_{\text {ice }}=1.31$ and the value $\rho_{\text {ice }}=0.9216 \mathrm{~g} / \mathrm{cm}^{3}$ at the center of IceCube [19]. This introduces a small systematic uncertainty of about $1 \%$, which can be corrected for by rescaling our results to the correct density.
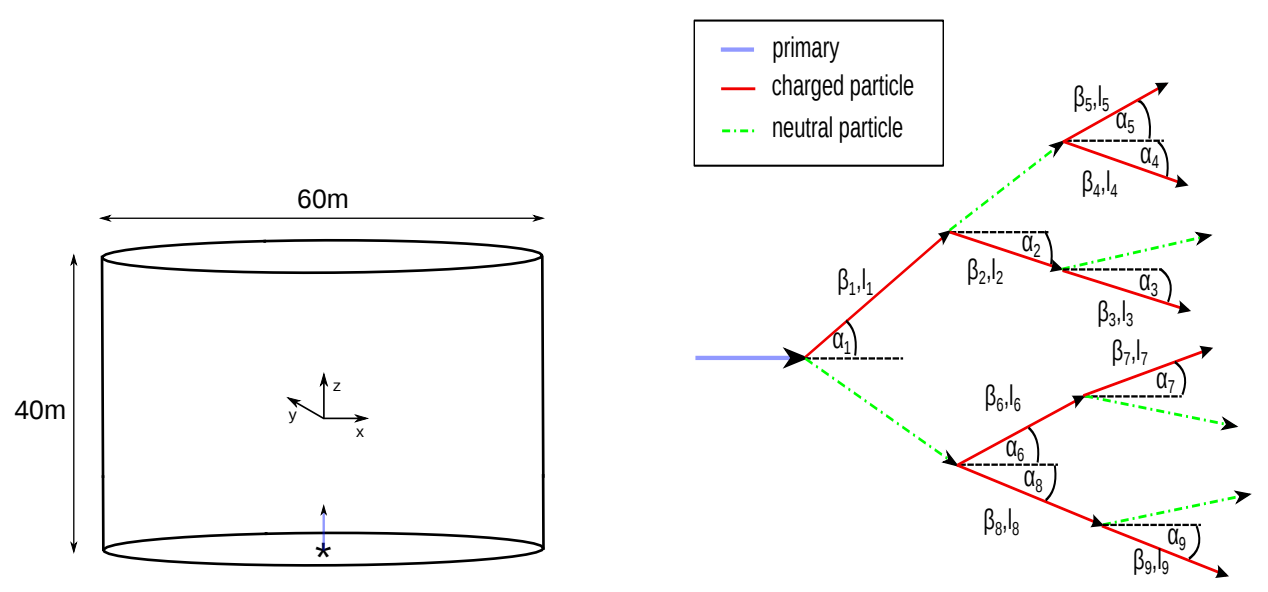

Figure 2: Geometry of the simulation and method of the calculation

The simulation principle of this work is illustrated in figure 2. The medium is contained in a cylindrical volume of $30 \mathrm{~m}$ radius and $40 \mathrm{~m}$ height. The dimensions are chosen such that all secondary particles are well confined within the geometry and fully tracked. The primary particle $e^{ \pm}$or $\gamma$ is injected at the bottom center into this volume with its initial momentum pointing into positive z-direction. The particles are propagated through the medium and secondary particles are created, which again can produce further particles. Each step between two interactions corresponds to a track segment for which the energy and direction are assumed constant. For each track segment $i$ we store the length $l_{i}$, the Lorentz factor $\beta_{i}$, the 
$z$-position $z_{i}$ and the direction $\alpha_{i}$ with respect to the z-axis. The azimuth angle $\phi$ discussed in section 3.6 corresponds to the rotation angle in the $x-y$ plane. Summing over all track segments allows to calculate the Cherenkov photon yield and the corresponding angular distribution.

For the here described simulation it is important to simulate all particles with energies above the Cherenkov-threshold. Details on the simulated physics processes are given in Appendix A. In Geant 4 some electromagnetic processes require production thresholds to avoid infrared divergences [20]. These production thresholds are specified as a cut-in-range threshold, using the SetCuts () method of G4VUserPhysicsList. Here, particles are tracked if their mean expected range is larger than this cut-in-range threshold. For each material and particle type, this cut-in-range is transformed into a corresponding energy threshold. Here, a cutin-range of $100 \mu \mathrm{m}$ is chosen. This corresponds to a kinetic energy threshold of $E_{\text {cut }, e^{ \pm}} \approx 80 \mathrm{keV}$ for electrons, which is well below the Cherenkov-threshold $E_{\mathrm{c}, e^{ \pm}} \approx 264 \mathrm{keV}$. Once produced, all secondary particles are tracked until they stop. In order to increase the computing performance, a single scattering process of a particle does not correspond to an individual track segment but multiple scattering processes are simulated as one step.

We perform simulations up to a maximum energy of the primary particle of $10 \mathrm{TeV}$ to which electromagnetic cross sections in Geant 4 are valid. Our results could be extrapolated beyond this limit, however, at high energies an additional effect, the LPM effect, is expected to set in. This effect describes significantly reduced electromagnetic cross sections and the longitudinal development of such cascades would become strongly elongated such that our parameterization approach is not valid.

For $\beta=1$, the number of emitted Cherenkov-photons is proportional to the length of the track and can be calculated using Eq.(2). For $\beta<1$ the photon yield is smaller and proportional to the factor

$$
\sin ^{2}\left(\theta_{c}\right)=1-\cos ^{2}\left(\theta_{c}\right)=1-\frac{1}{\beta^{2} \cdot n^{2}} .
$$

In order to properly account for this smaller yield, the length of each track segment $l$ is scaled with the Frank-Tamm factor

$$
\hat{l}=\frac{\sin ^{2}\left(\theta_{c}\right)}{\sin ^{2}\left(\theta_{c, 0}\right)} \cdot l \quad \text { with } \quad \sin ^{2}\left(\theta_{c, 0}\right)=1-\frac{1}{n^{2}} .
$$

The value $\hat{l}$ thus corresponds to the equivalent length of a relativistic track with the same photon yield as the track length $l$. The use of the equivalent length $\hat{l}$ instead of an explicit calculation of photons has the advantage that the here presented results 
can be rescaled to slightly different indices of refraction, and are independent of the assumed wavelength interval of the considered photo-detector.

The angular distribution of the Cherenkov-photons is calculated with the method introduced in [18]. In this method the distribution of track length as a function of the directional angle $\alpha$ with respect to the z-axis (zenith) and the velocity $\beta$ can be transformed to a zenith distribution of emitted Cherenkov-photons. The prerequisite for the applicability of that method is a high statistics of tracks which are distributed uniformly in azimuth.

\section{Results}

\subsection{Velocity distribution of shower particles}

Figure 3 shows the distribution of track length in the cascade versus the Lorentz factor $\beta$ for different primary energies $E_{0}$. The shape is remarkably constant for different $E_{0}$ while the total normalization is proportional to $E_{0}$. The distribution also does not depend on the type of the primary particle. The difference between the physical track-length $l$ and the effective track length $\hat{l}$ becomes particularly obvious close to the Cherenkov-threshold $\beta \approx 0.752$. Unless noted otherwise, we will use in the following the effective track length $\hat{l}$ instead of the physical track length $l$.

\subsection{Total light yield}

Figure 4 (top) shows as an example the distribution of the total effective track length for repeated simulations of a primary electron of $1 \mathrm{TeV}$. The distribution can be well described by a Gaussian, which is fit to the data.

The mean expectation and the standard deviation from Gaussian fits to distributions for different primary particles and different primary energy $E_{0}$ are shown in figure 4 (middle) and (bottom). The data is fit with a power-law

$$
\hat{l}\left(E_{0}\right)=\alpha \cdot E_{0}^{\beta} \quad, \quad \sigma_{\hat{l}}\left(E_{0}\right)=\alpha \cdot E_{0}^{\beta} .
$$

In all fits the parameter $\beta$ is found to be consistent with 1 at the level $10^{-5}$ indicating a very good linear relation between the total $\hat{l}$ and $E_{0}$. Also the coefficients $\alpha$ agree within $10^{-3}$ for different primary particles. The detailed results of these fits are given in table B.3 in Appendix B. As a result we obtain an energy scale parameter which relates linearly the total Cherenkov-light yield with the primary energy

$$
\alpha \approx 532.1 \pm 10^{-3} \mathrm{~cm} \mathrm{GeV}^{-1} .
$$

The observed value $\alpha=532.1 \mathrm{~cm} \mathrm{GeV}^{-1}$ is slightly larger than the value $\alpha=$ $521 \mathrm{~cm} \mathrm{GeV}^{-1}$ in [12] which was also obtained in Geant 4 simulations of ice. The 

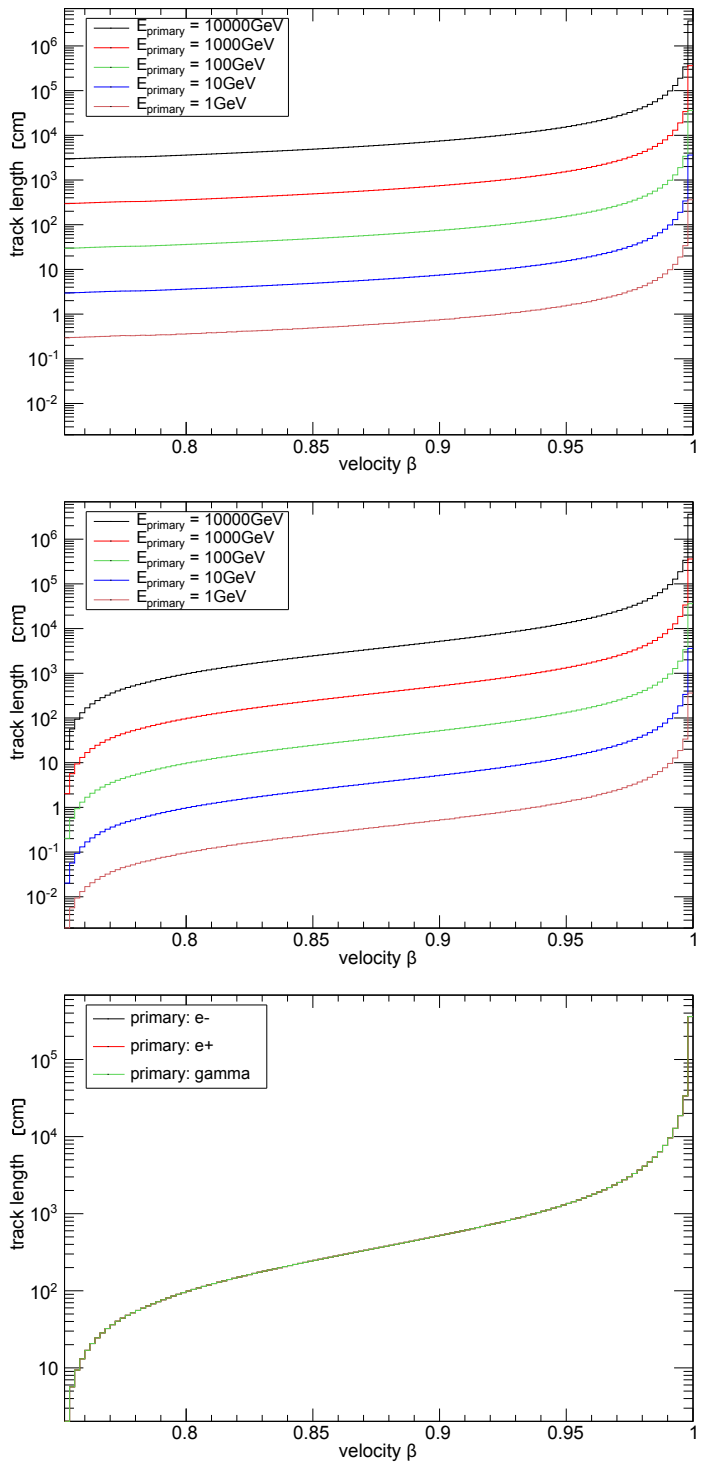

Figure 3: Velocity distribution of track length. Shown is the differential distribution of summed track length per shower versus the Lorentz factor $\beta$ for bins of 0.002 in $\beta$. The top figure show the distributions of physical length $l$ for the shower from primary positrons of different energies. The middle figure shows the same distribution for the track length $\hat{l}$ which has been weighted with the Frank-Tamm factor, Eq. 6). The bottom figure shows the distributions of $\hat{l}$ for different primary particles: $e^{+}, e^{-}, \gamma$ for the primary energy $E_{0}=1 \mathrm{TeV}$. 

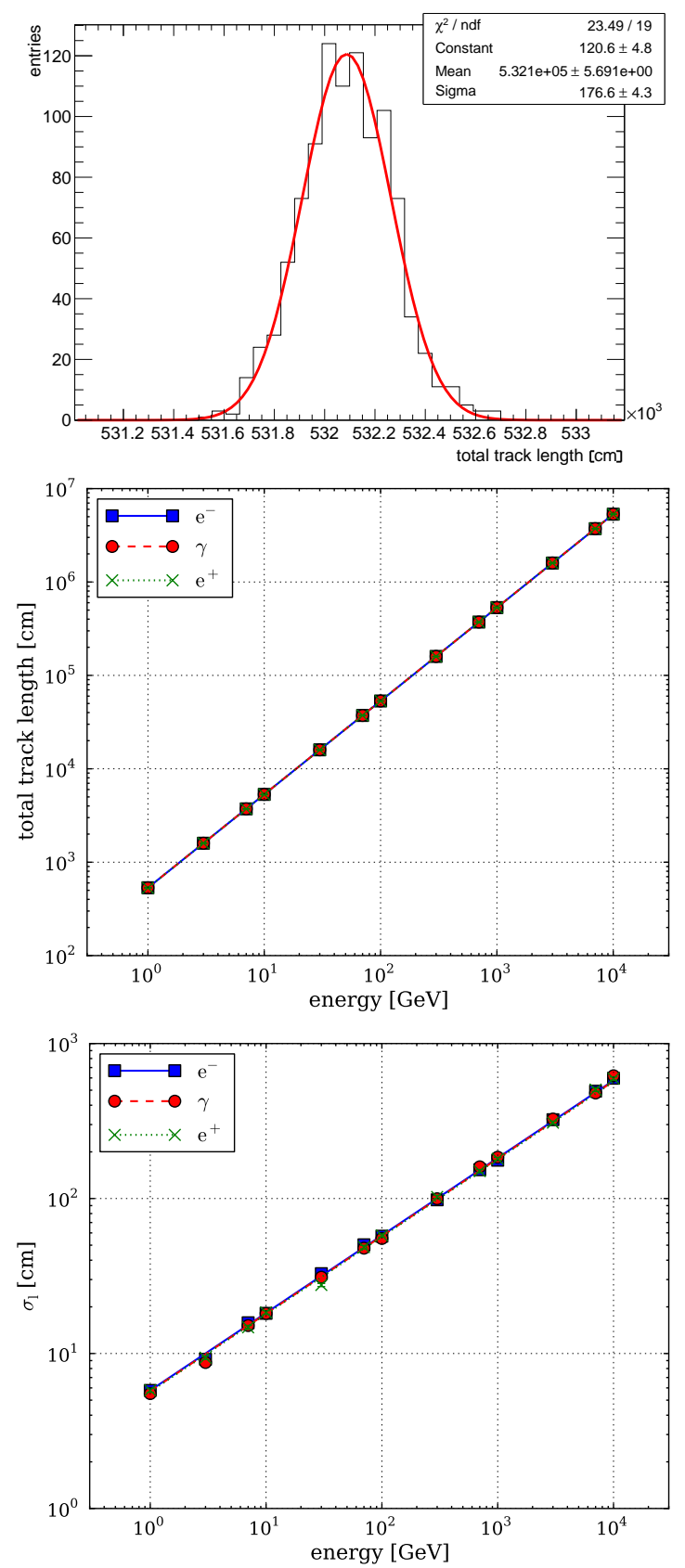

Figure 4: Total amount of Cherenkov-light-radiating track length $\hat{l}$, including the Frank-Tamm factor. Top: distribution of $\hat{l}$ for $5 \cdot 10^{4}$ simulated primary electrons of $E_{0}=1 \mathrm{TeV}$. A Gaussian-distribution is fit to the data. Middle: $\hat{l}\left(E_{0}\right)$ as a function of the primary energy $E_{0}$ resulting from Gaussian fits. A power-law (Eq. (7) is fit to the data. Bottom: Standard deviation $\sigma_{\hat{l}}\left(E_{0}\right)$ Resulting from Gaussian fits. A power-law (Eq. (7p) is fit to the data. 
origin of this $2 \%$ difference is not obvious. It could be either related to difference in the versions of Geant 4 but also to different configurations which are not given in [12], e.g. a slight difference in the assumed index of refraction.

If rescaled to the density of water by the relation

$$
\rho_{\text {water }} \cdot \alpha_{\text {water }} \approx \rho_{\text {ice }} \cdot \alpha_{\text {ice }}
$$

we obtain the value $\alpha_{\text {water }} \approx 484 \mathrm{~cm} \mathrm{GeV}^{-1}$. This is significantly larger than the values $437 \mathrm{~cm} \mathrm{GeV}^{-1}$ found in [11] for $n=1.33$ and $466 \mathrm{~cm} \mathrm{GeV}^{-1}$ in [14] for $n=1.35$.

As expected for an increasing number of particles, the size of fluctuations of the total track length $\sigma_{\hat{l}}$ increases $\propto \sqrt{E_{0}}$ and the value $\beta=0.5$ is fixed for the fit. Hence, the relative size of fluctuation decrease $\propto 1 / \sqrt{E_{0}}$ with higher primary energy $E_{0}$ (see figure 4). With the values in table B.3 in Appendix B the relation

$$
\frac{\sigma_{\hat{t}}}{\hat{l}} \approx 0.0108 \cdot \sqrt{\frac{1 \mathrm{GeV}}{E_{0}}}
$$

is found.

\subsection{Longitudinal cascade development}
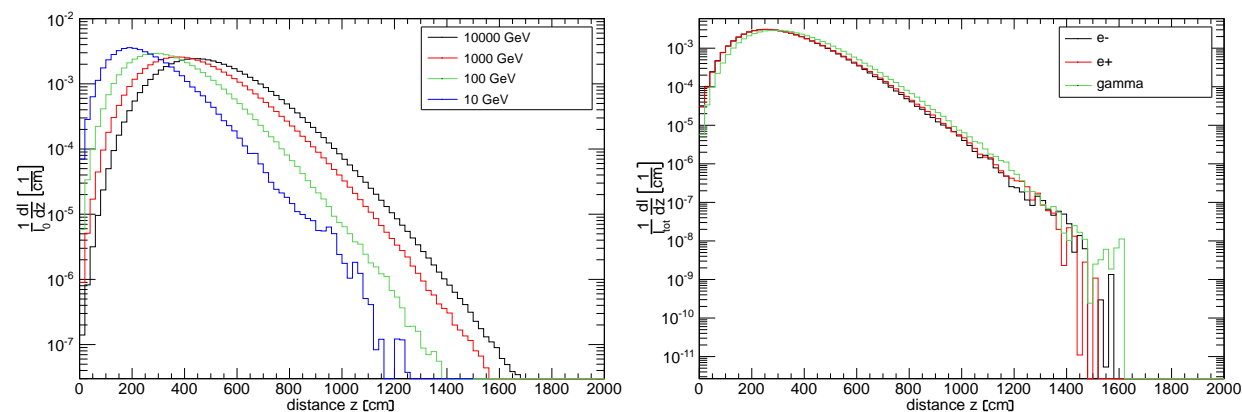

Figure 5: Longitudinal shower profiles as a function of the length $z$ along the shower axis. Shown is the track length distribution $\frac{d \hat{l}}{d z}$ relative to the total length $\hat{l}_{0}$ of the cascade. The left figure shows the result for initial $\gamma$ and different primary energies $E_{0}$. The right figure shows the result for different primary particles $e^{ \pm}, \gamma$ and a primary energy $E_{0}=100 \mathrm{GeV}$. All track segments have been weighted with the Frank-Tamm factor.

The longitudinal profiles of the track length $d \hat{l} / d z$ along the axis of the cascade are shown in figure 5. As expected from a simple Heitler model [6, 9], the depth of the shower maximum $z_{\max }$ scales logarithmically with the primary energy. The 
distributions are almost identical for $e^{+}$and $e^{-}$. However, for an initial photon the depth of the shower maximum is about one radiation length deeper.

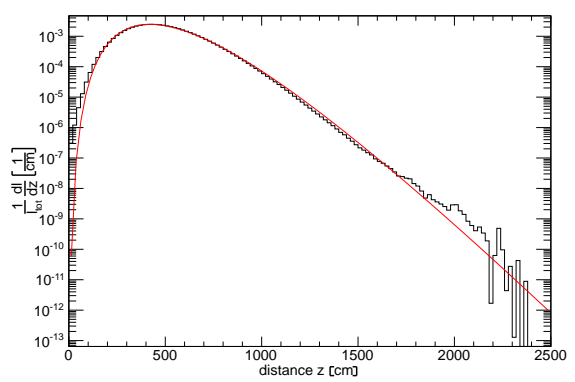

Figure 6: Example of the parameterization of the longitudinal shower profile with Eq. (11) for a positron with a primary energy $E_{0}=10 \mathrm{TeV}$.

The longitudinal shower profile can be parameterized with a gamma distribution

$$
\widehat{l}_{\mathrm{tot}}^{1} \cdot \frac{\widehat{\mathrm{d} l}}{\mathrm{~d} t}=b \frac{(b t)^{a-1} \mathrm{e}^{-b t}}{\Gamma(a)} .
$$

Here, $t$ is the shower depth $t \equiv z / X_{0}, a$ and $b$ are characteristic dimensionless constants [6]. An example fit is shown in figure 6, The results of all fits for different $E_{0}$ are given in table B.7 in Appendix B.

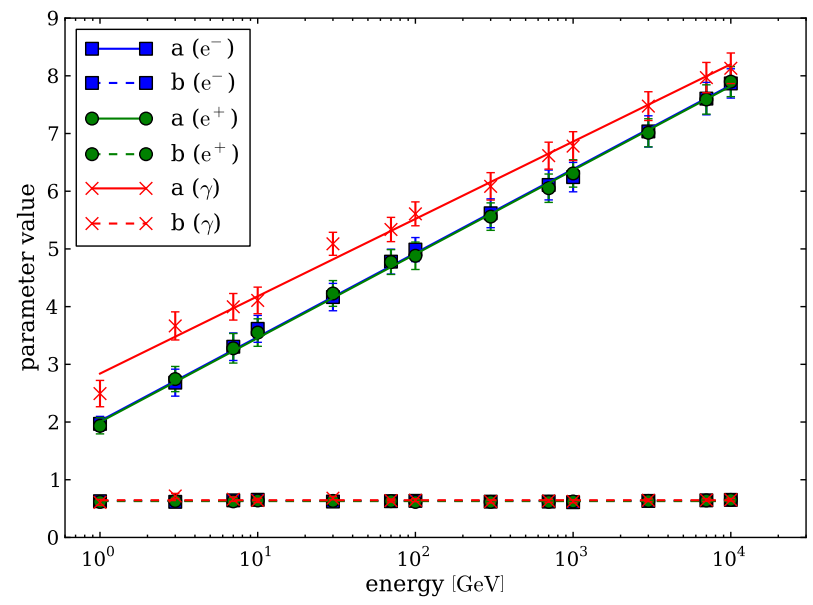

Figure 7: Fit parameters of the longitudinal profile $a, b$ versus initial energy.

The energy dependence of the fit parameters $a$ and $b$ is shown in figure 7, $b$ is found constant and does not depend on the particle type, while the parameter $a$ can 
be described with an logarithmic increase

$$
a=\alpha+\beta \cdot \log _{10}\left(\frac{E_{0}}{1 \mathrm{GeV}}\right) .
$$

It is slightly larger for $\gamma$ than for $e^{ \pm}$. The parameterization results for $b$ and $\alpha, \beta$ are given in table B.4 in Appendix B.

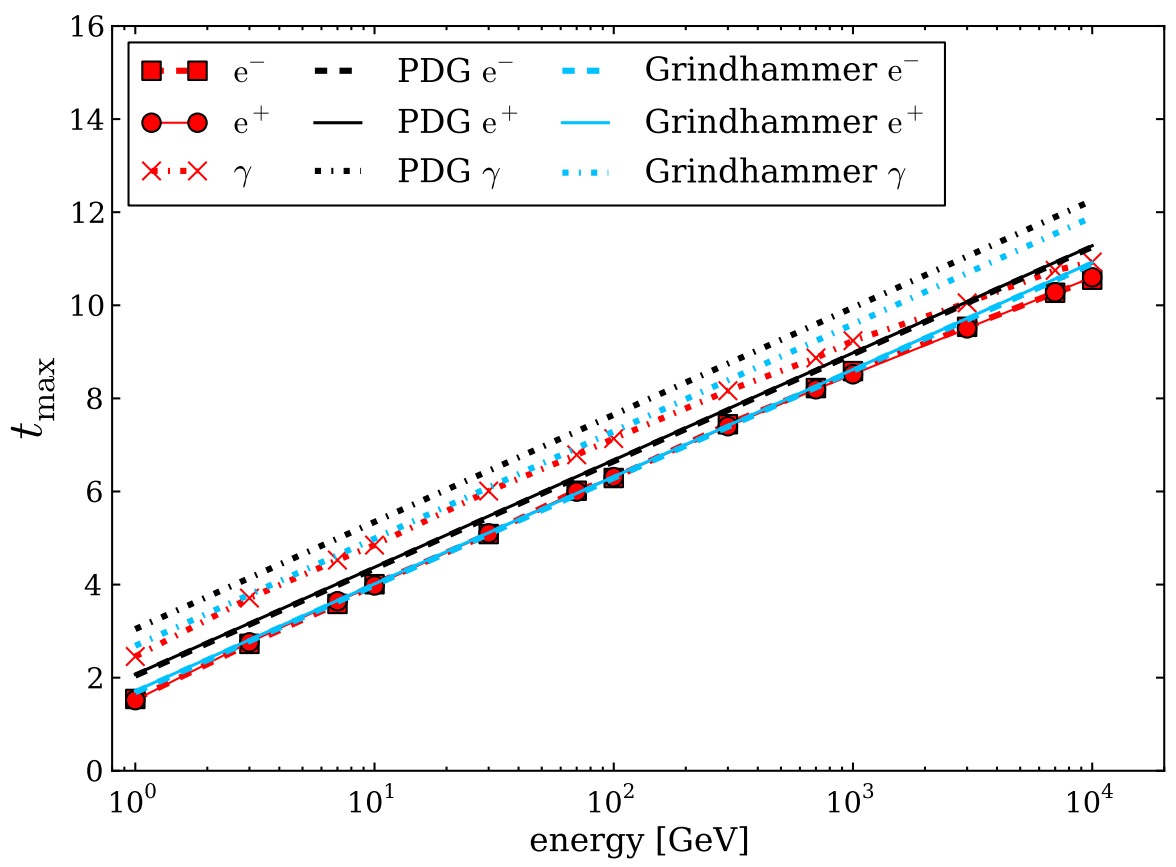

Figure 8: Maximum of the longitudinal Cherenkov-radiating track-length profile as a function of the initial energy. The markers represent the calculated values for $t_{\max }$ and solid lines are for visual guidance. The dotted/dashed lines show Eq.(14) with the parameters from [6] (PDG)/[21](Grindhammer) respectively.

Figure 8 shows the shower maximum of the Cherenkov-radiating track length as a function of the initial energy. It has been calculated from the longitudinal shower profiles with the formula

$$
t_{\max }=\frac{a-1}{b} .
$$

Additionally shown is the maximum of the longitudinal energy deposition based on the parameterization in [6]

$$
t_{\max }=\ln y+C_{\mathrm{j}}, \quad \mathrm{j}=\mathrm{e}, \gamma,
$$


with $y=E_{0} / E_{\text {crit }}$. In [6] for electron- or positron-induced cascades the values $C_{\mathrm{e}}^{\mathrm{PDG}}=-0.5$ and for photon-induced cascades $C_{\gamma}^{\mathrm{PDG}}=+0.5$ are given. These values are based on simulations with EGS4 up to an energy of $100 \mathrm{GeV}$ for nuclei heavier than carbon. Up to that energy the slope agrees with our result but our values are offset by about -0.5 . Above $100 \mathrm{GeV}$ we also deviate in slope.

In contrast, [21] gives the value $C_{\mathrm{e}}^{\mathrm{Gr}}=-0.858$ for electron- or positroninduced cascades. This parameter was also obtained from fits to the longitudinal energy deposition profiles for elements ranging from carbon to uranium at energies from $1 \mathrm{GeV}$ to $100 \mathrm{GeV}$. The simulations were performed with Geant3. The value $C_{\gamma}^{\mathrm{Gr}}$ is not explicitly stated. Assuming that the difference of the maxima of photon- and electron-induced cascades is about one radiation length we obtain $C_{\gamma}^{\mathrm{Gr} .} \approx C_{\mathrm{e}}^{\mathrm{Gr} .}+1=+0.142$.

Here, the simulations are performed with Geant 4 and ice is used as the detector material. It can be seen that our results agree much better with [21]. Nevertheless, deviations appear for larger energies, where the here obtained energy dependence increases less than logarithmically. This effect is stronger for photon-induced cascades than for electron-induced cascades.

\subsection{Angular distribution of tracks}
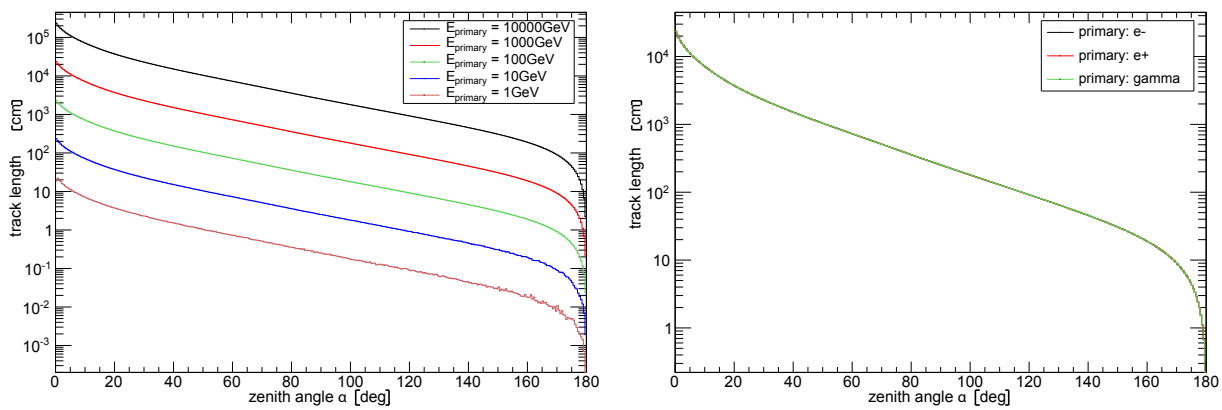

Figure 9: Distribution of the effective track length $\hat{l}$ per shower as a function of the inclination angle $\alpha$ of the primary particle's direction. The left figure shows the result for a primary positron and different $E_{0}$ and the right figure the results for $E_{0}=1 \mathrm{TeV}$ and different primary particles.

Relevant for the angular distributions of Cherenkov-photons is the angular distribution of secondary tracks in the cascade and their velocity $\frac{d^{2} \hat{l}}{d \alpha d \beta}$. Here, $\alpha$ is the polar angle of the track with respect to the $z$ axis and $\beta$ the Lorentz-factor.

The distribution $\frac{d \hat{l}}{d \alpha}$ is shown in figure 9 and the distribution $\frac{d^{2} \hat{l}}{d \alpha d \beta}$ in figure 10

Most particles are produced in forward direction with a velocity $\beta$ close to 1 . It can be seen that the normalization of the $\alpha$-distribution changes with energy but 


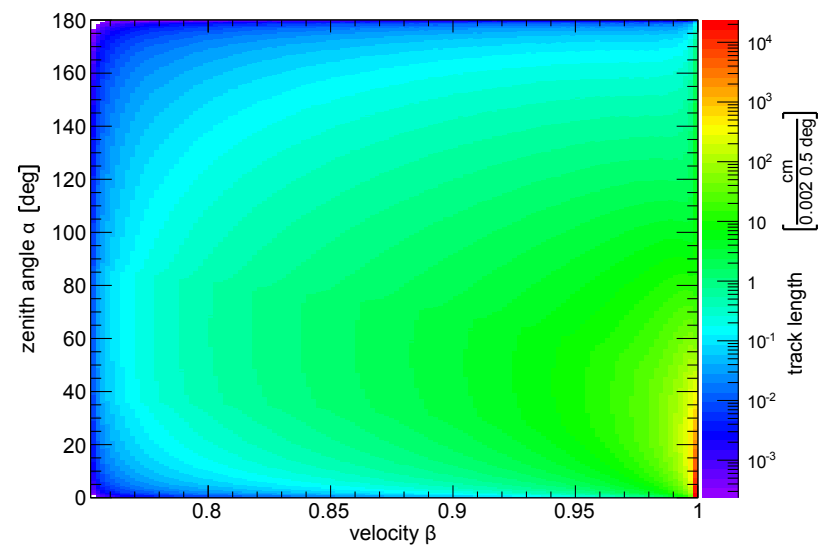

Figure 10: Density distribution of the effective track length $\hat{l}$ versus the inclination angle $\alpha$ and Lorentz factor $\beta$ for a $1 \mathrm{TeV}$ shower. The vertical color codes corresponds to the histogrammed length $\hat{l}$ per shower.

the shape does not. The angular distribution does not change for different primary particles.

\subsection{Angular distribution of Cherenkov-light}
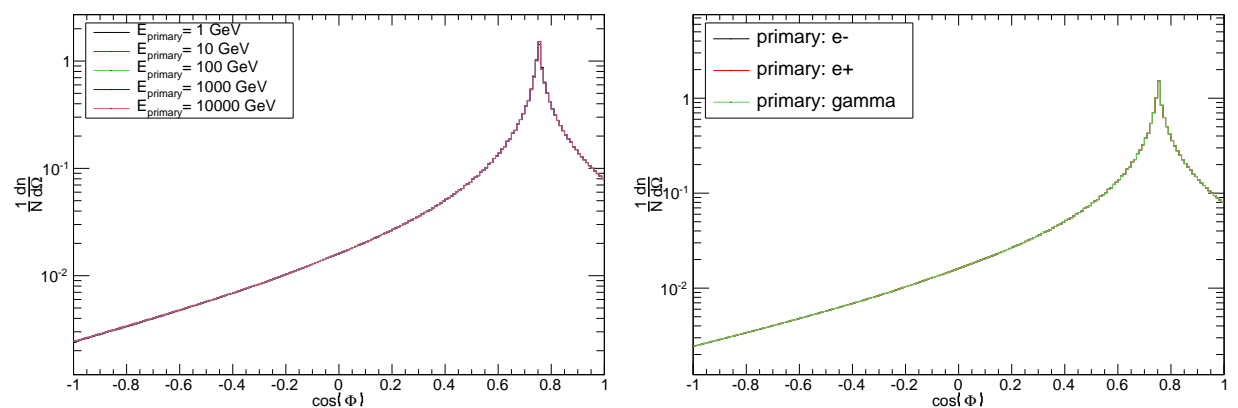

Figure 11: The angular distribution of Cherenkov photons for different $E_{0}(\mathrm{left})$ and different primary particles (right). Shown are the normalized angular distributions per photon and steradian.

The angular distribution of Cherenkov-photons is calculated with the method described in [18]. Figure 11 shows example distributions $\frac{d \hat{l}}{d \Phi}$ versus the zenithal 
angle $\Phi$ with the z-axis 1 . The normalization of the distribution corresponds to the track length that produces an equivalent total Cherenkov light yield.

A broad distribution with a clearly pronounced Cherenkov peak is visible. As expected from the results in section 3.4, the shape of the distribution is unchanged for different primary energies and different primary particles.

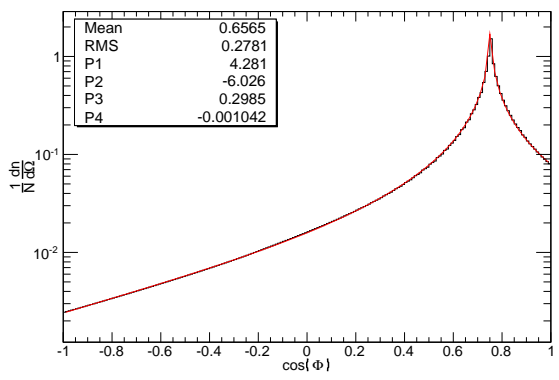

Figure 12: Example of the parameterization of the angular distribution for a $E_{0}=$ $100 \mathrm{GeV}$ positron. The parameters $P 1$ to $P 4$ correspond to the parameters $a$ to $d$ in Eq.(15).

The angular distributions are parameterized with a simple function

$$
\frac{d n}{d \Omega}=a e^{b\left|x-\cos \Theta_{c, 0}\right|^{c}}+d .
$$

A typical fit is shown in figure 12 .

The fit parameters for different energies are given in table B.9 in Appendix $B$. They are found to be very similar and constant with energy. We conclude that the angular distribution of Cherenkov-photons can be described with the above formula and the averaged parameters given in table B.5 in Appendix B

\subsection{Fluctuations in azimuth}

An important prerequisite for the here used calculation of the angular distribution of Cherenkov-photons is the assumed symmetry in azimuthal angle $\phi$ of the distribution of track directions $\mathrm{s}^{2}$ in the plane around the shower axis [18]. As an example, figure 13 shows azimuthal distributions of $\hat{l}$ for four individual showers, each for two different energies. Differences originate from fluctuations in the

\footnotetext{
${ }^{1}$ Note that the definition of $\Phi$ is identical to the previously defined angle $\alpha$. However we use a different symbol to indicate the difference of photons and tracks.

${ }^{2}$ Note, that the azimuthal angle $\phi$ is different from the earlier defined angle $\Phi$ (see section 3.5 . which was the zenithal emission angle of Cherenkov-photons.
} 

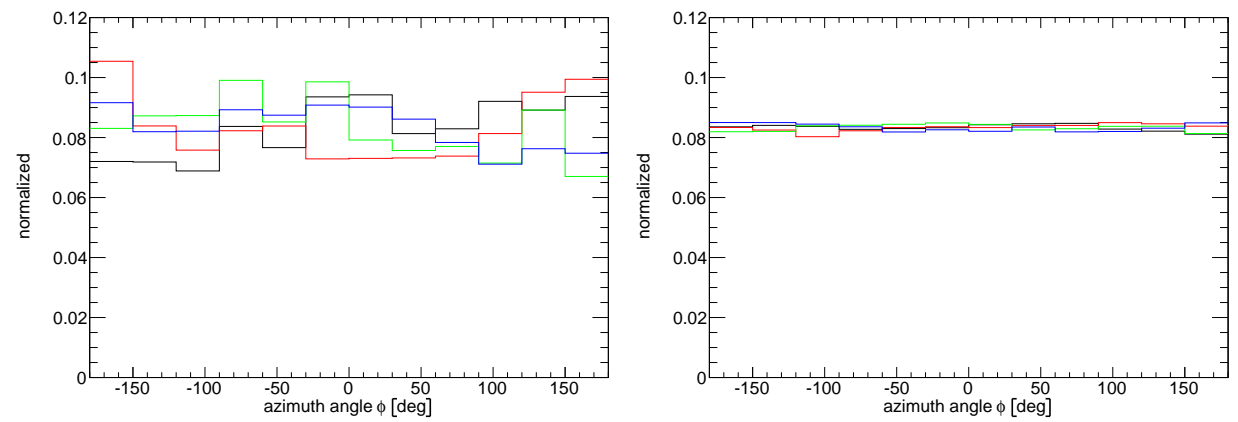

Figure 13: Example of the azimuthal distribution of four individual showers of electrons with a primary energies $E_{0}=10 \mathrm{GeV}$ (left) and $E_{0}=1 \mathrm{TeV}$ (right). The relative effective track length per $30^{\circ}$-interval is shown.

shower development. Correspondingly, the relative size of fluctuations strongly decreases for larger particle numbers in higher energy showers and the distribution becomes almost flat in azimuth.

The effect of this asymmetry can be quantified by aligning all simulated showers in azimuth in order to account for their random orientation. To obtain an averaged azimuthal distribution the bin contents for each shower are added with the maximum bin aligned and the azimuthal orientation is defined according to the direction of the second highest bin. The results are shown in figure 14. The mean total amplitude of angular fluctuations in azimuth can be as large as $\pm 11 \%$ for $10 \mathrm{GeV}$ but decreases approximately with the square root of the primary energy to less than $\pm 1.1 \%$ for $E_{0}=1 \mathrm{TeV}$. However, as indicated by the error bars, the individual bin fluctuations are of the same order of magnitude as the mean amplitude of the asymmetry.

The amplitude of the asymmetry is fit with the parabolic function

$$
A(\phi)=\frac{1}{12}\left[1+b\left\{\left(\phi-\phi_{0}\right)^{2}-\frac{1}{6 \pi}\left(\left(2 \pi-\phi_{0}\right)^{3}+\phi_{0}^{3}\right)\right\}\right],
$$

The parameter $b$ describes the vertical compression and $\phi_{0}$ the position of the minimum. The third term accounts for the normalization. The angle $\phi$ is used in units of radians and the bin size of the histogram is $\Delta \phi=30^{\circ}=0.523 \mathrm{rad}$. The results of all fits are given in table B.10 in Appendix B.

The parameter $\phi_{0}$ is found roughly constant. It differs slightly from $\pi$ because the preferential direction of the second largest bin leads to an angular bias. The energy dependency is shown in figure 14 (bottom right). The amplitude coefficient $b$ is fit with

$$
b=\frac{p}{\sqrt{E_{0}}}
$$



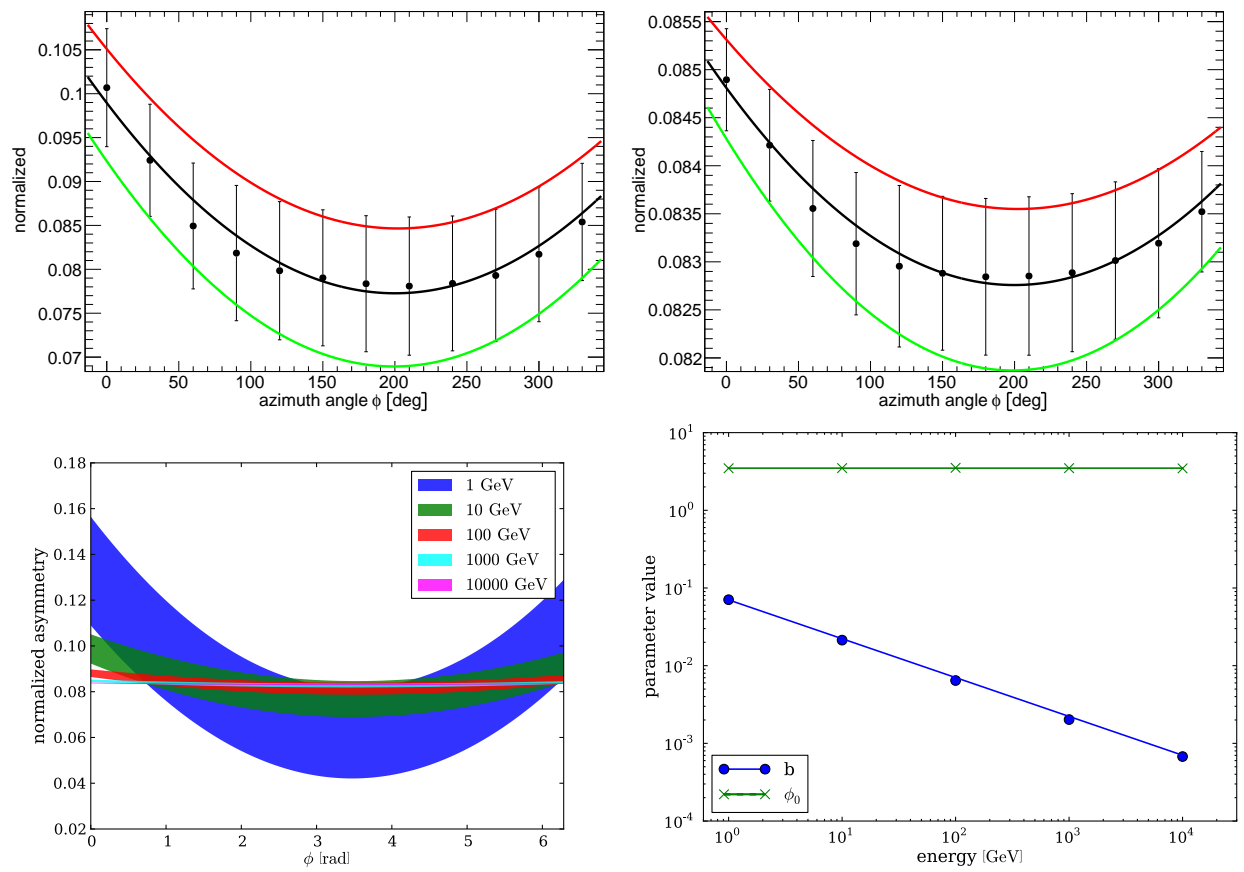

Figure 14: Fitted shower asymmetry of electron-induced showers for different primary energies: $E_{0}=10 \mathrm{GeV}$ (top left) and $E_{0}=1 \mathrm{TeV}$ (top right). The dots correspond to the mean expectation, if each shower is aligned before averaging as described in the text. The shown error bars represent the standard deviation of the individual bins. A parabola Eq. 16 was fit to the distributions. The black curve represents the fit to the data and the red and green curves fits, where each bin content was increased or reduced by one standard deviation. The bottom left figure shows the range of the fitted uncertainties for different primary energies, and the bottom right figure the results of the parameters in Eq.(16) versus the primary energy.

as expected from the correspondingly increased number of particles in the cascade. The results of these fits are summarized in table B.11 in appendix Appendix B.

\subsection{Dependence of the angular distribution on the shower age}

The shape of the angular distribution of tracks $d \hat{l} / d \alpha$ and therefore the angular distribution of Cherenkov-light has been found to be independent of the energy $E_{0}$. However, the electromagnetic cascade has an extension of a few meter (see section 3.3. Within this evolution of the cascade it is plausible that large scattering angles occur more frequently at later stages of the shower development than at earlier. 
Therefore, we investigate how the angular distribution of tracks changes with the age of the shower.

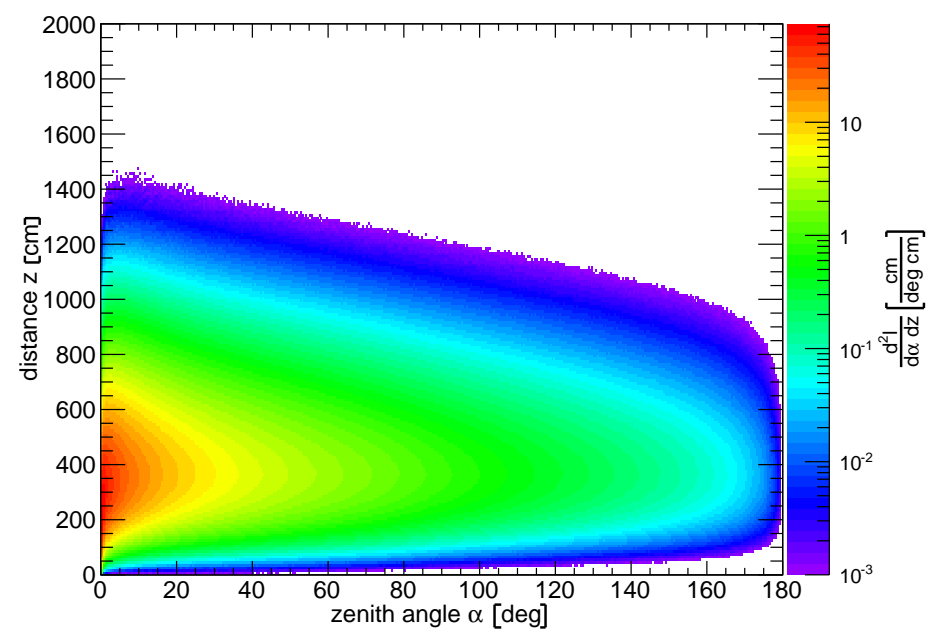

Figure 15: Distribution of the relative track length versus the shower length $z$ and the inclination angle $\alpha$ for a $1 \mathrm{TeV}$ electron shower. The vertical color code corresponds to the histogrammed lengths $\hat{l}$ normalized per initial particle.

Figure 15 shows the angular distribution of the track length density versus the longitudinal length of the shower $\frac{d^{2} \hat{l}}{d \alpha d z}$. The distribution is found to be largely dominated by the longitudinal evolution of the particle density and only a small difference of the angular distribution between the onset and the end of the cascade can be seen.

For a more detailed investigation we split cascades along the shower axis $z$ into parts of different shower age. We chose three slices such that they contain the same total track length and thus emit the same total amount of Cherenkov-light.. The resulting angular distributions are shown in figure 16. Large differences are only seen in the very forward region $\alpha<10^{\circ}$. With increasing shower age the track length becomes larger by about a factor 3 . The differences for large angles $\alpha>20^{\circ}$ are comparably smaller, with about $10 \%$ change in yield and no obvious change in shape. The situation is similar for higher energy $E_{0}$.

The resulting distributions of Cherenkov-photons are shown in figure 17. The large opening angle of the Cherenkov cone leads to a substantial smearing of the angular distribution. Hence, the relatively strong effect into the forward direction for the angular track length distribution does not propagate to an equal strong variation of the Cherenkov peak. Here, only an effect of $\pm 10 \%-20 \%$ in the variation of the peak is observed. 

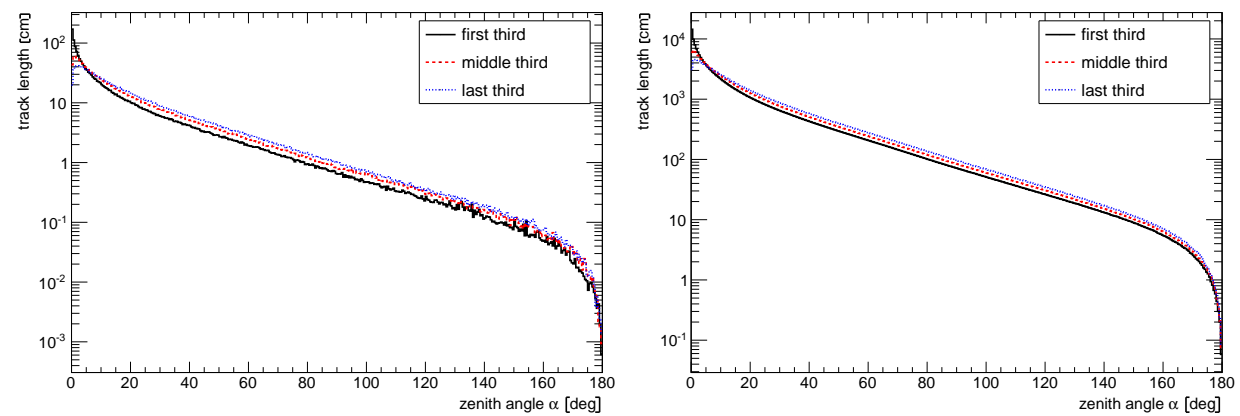

Figure 16: Angular distributions of the track length $\hat{l}$ for different slices of the longitudinal shower evolution. The figures show the histograms for a primary $\gamma$ with energy $E_{0}=10 \mathrm{GeV}$ (left) and $E_{0}=1 \mathrm{TeV}$ (right) normalized to the track length per particle. The longitudinal distribution has been split into 3 slices of equal total track length.
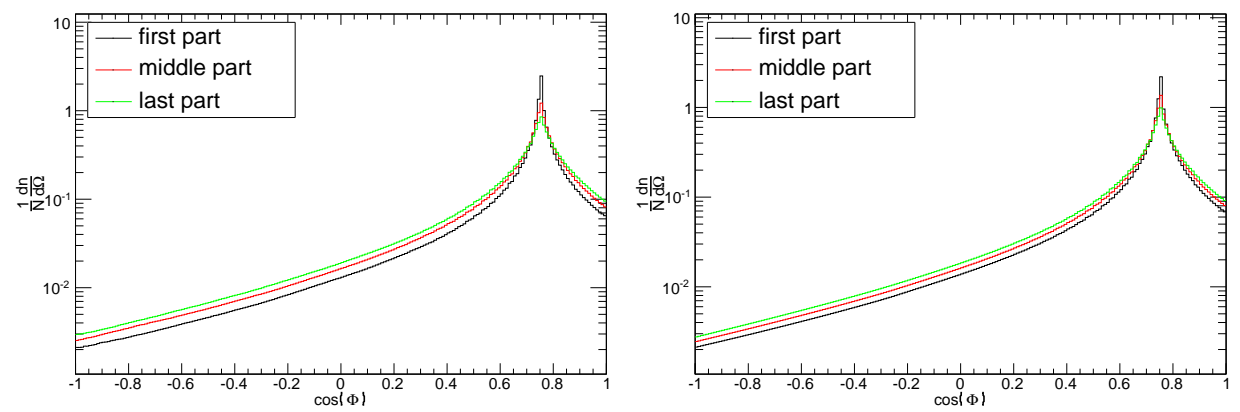

Figure 17: Angular distribution of the emitted Cherenkov-photons for the different slices of the longitudinal shower evolution. The figures show the histograms for a primary $\gamma$ with energy $E_{0}=10 \mathrm{GeV}$ (left) and $E_{0}=1 \mathrm{TeV}$ (right) normalized to the track length per particle. The longitudinal distribution has been split into three slices of equal track length.

In summary, we find that the differential variation of Cherenkov-light emission along the length of the cascade is a relatively weak effect. A global angular distribution, as parameterized in section 3.5, seems justified in particular when considering that the length of a cascade is short compared to the typical spacing of optical sensors in neutrino telescopes. However, for more detailed information we have repeated the angular parameterization Eq. 15 also for the three different slices in shower age separately. The results of the parameterization are given in table B.13, B.15 and B.15 in Appendix B 


\section{Summary and Conclusions}

We have simulated electromagnetic cascades with Geant 4 for different primary particles and primary energies $E_{0}$. We have parameterized the total Cherenkovlight-radiating track length and its fluctuations, the longitudinal development of the cascade and the angular distribution of emitted Cherenkov-photons.

Our result for the total track length agrees within $2 \%$ with the result obtained in [12] but disagrees with other previous calculations. The relative size of fluctuations for different showers decreases $\propto 1 / \sqrt{E_{0}}$.

The longitudinal profiles are found to be well described by a gamma distribution and a difference between $e^{ \pm}$and $\gamma$ is observed as expected [6]. However, quantitatively the position of the shower maximum deviates from the values in [6], but agrees with [21]. For higher energies than $100 \mathrm{GeV}$ we observe a change of slope in the elongation rate.

The angular distribution of tracks in the cascade and the corresponding distribution of photons is found to be independent of $E_{0}$ and the type of primary particle.

Systematic uncertainties of our parameterizations are related to the used refraction index and density of ice. They are of the order of $1 \%$ for typical values of ice and can be corrected, e.g. by rescaling the observables of our parameterizations which depend on length scales such as the axis of the shower development to the correct media density and by using the correct Cherenkov angle.

Also uncertainties of the used differential cross sections of electromagnetic processes in Geant 4 can reach up to a few percent but are generally substantially smaller [22]. We do not expect these differential uncertainties to significantly affect our global results, particularly the total track length is not strongly affected. Hence we estimate a typical uncertainty of less than $1 \%$.

The LPM effect and dielectric suppression [23] are considered in the used version of Geant4 [24] but are only expected to become significant at larger energies than considered here. Electronuclear interactions have not been simulated, because their cross section is small.

The fluctuations of the total track length for different individual showers are found to decrease with $\propto 1 / \sqrt{E_{0}}$ and the related uncertainty is already smaller than $1 \%$ for a few $\mathrm{GeV}$.

For the determination of the uncertainties in the calculation of the angular distribution of emitted Cherenkov-light we investigate and parameterize the uncertainty related to azimuthal fluctuations and the evolution of the angular distribution with increasing shower age. The azimuthal asymmetry of tracks is found to be small (about $7 \%$ at $1 \mathrm{GeV}$ ) and to decrease $\propto 1 / \sqrt{E_{0}}$. When taking into account the large emission angle of Cherenkov-photons this effect is expected to be further washed out for the angular distribution of Cherenkov-light and is largely negligible 
at high energies. Also the effect of the longitudinal shower evolution is small and results in differences in the width of the Cherenkov peak in the angular distribution. This effect becomes even less important for distances larger than the scale length of the cascade and it is washed out e.g. by the scattering of photons when propagating through the medium.

\section{Appendix A. Geant4 configuration parameters used for this study}

In this chapter a summary of the defined media properties and physics processes is given.

Appendix A.1. Materials

\begin{tabular}{|c|c|c|c|c|}
\hline Medium & Density $\frac{\mathrm{g}}{\mathrm{cm}^{3}}$ & Index of refraction & Element & Fraction of mass \\
\hline \multirow[t]{2}{*}{ Ice } & 0.910 & 1.33 & Hydrogen & $88.81 \%$ \\
\hline & & & Oxygen & $11.19 \%$ \\
\hline
\end{tabular}

Table A.1: Composition of ice as used in the Geant4-simulation.

In Geant 4 macroscopic properties of matter are described by G4Material and the atomic properties are described by G4Element. A material can consist of multiple elements and therefore represent a chemical compound, mixture as well as pure materials. For the performed simulations ice was used. Unless noted otherwise, the value $n=1.33$ is used for the index of refraction. The simulated properties of ice are summarized in table A.1.

\section{Appendix A.2. Physicslist}

All physics processes, which are used during the simulation must be registered in G4VUserPhysicsList. These simulations are based on the standard physics list G4EmStandardPhysics_option3. The included processes are summarized in table A.2. The maximum energy for the cross section tables and the calculation of $\mathrm{d} E / \mathrm{d} x$ in Geant 4 is $10 \mathrm{TeV}$. 


\begin{tabular}{lll}
\hline \hline Particle & Process & Model \\
\hline$\gamma$ & G4PhotoElectricEffect & G4PEEffectFluoModel \\
& G4ComptonScattering & G4KleinNishinaModel \\
& G4GammaConversion & \\
\hline$e^{-}$ & G4eMultipleScattering & \\
& G4eIonisation & \\
& G4eBremsstrahlung & \\
\hline$e^{+}$ & G4eMultipleScattering & \\
& G4eIonisation & \\
& G4eBremsstrahlung & \\
& G4eplusAnnihilation & \\
\hline$\mu^{+}, \mu^{-}$ & G4MuMultipleScattering & \\
& G4MuIonisation & \\
& G4MuBremsstrahlung \\
& G4MuPairProduction \\
& G4MuNuclearInteraction \\
& G4CoulombScattering \\
\hline$\pi^{+}, \pi^{-}, K^{+}, K^{-}, p^{+}$ & G4hMultipleScattering \\
& G4hIonisation \\
& G4hBremsstrahlung \\
& G4hPairProduction \\
\hline \hline all unstable particles & G4ionIonisation \\
& G4hMultipleScattering \\
\hline \hline
\end{tabular}

Table A.2: Physics processes of most important particles used in the simulation. If no model is specified the default model is used. For hadrons and ions that are not listed multiple scattering and ionization are defined. 


\section{Appendix B. Parameterization results}

\begin{tabular}{lcc}
\hline & fit of $\hat{l}\left(E_{0}\right)$ \\
particle & $\alpha / \mathrm{cm} \mathrm{GeV}^{-1}$ & $\beta$ \\
\hline$e^{-}$ & 532.07078881 & 1.00000211 \\
$e^{+}$ & 532.11320598 & 0.99999254 \\
$\gamma$ & 532.08540905 & 0.99999877 \\
\hline \multicolumn{3}{c}{ fit of $\sigma_{\hat{l}}\left(E_{0}\right)$} \\
$e^{-}$ & 5.78170887 \\
$e^{+}$ & 5.73419669 & 0.5 \\
$\gamma$ & 5.66586567 \\
\hline
\end{tabular}

Table B.3: Result of the parameterization of the effective track length versus primary energy (section 3.2). The top table gives the results of fits of Eq.(7) for the fits $\hat{l}\left(E_{0}\right)$ and the bottom the standard deviation $\sigma_{\hat{l}}\left(E_{0}\right)$ of the fluctuations.

\begin{tabular}{lccc}
\hline particle & $\alpha$ & $\beta$ & $b$ \\
\hline$e^{-}$ & 2.01849 & 1.45469 & 0.63207 \\
$e^{+}$ & 2.00035 & 1.45501 & 0.63008 \\
$\gamma$ & 2.83923 & 1.34031 & 0.64526 \\
\hline
\end{tabular}

Table B.4: Results of the energy dependence of the longitudinal fits, section 3.3

\begin{tabular}{lcccc}
\hline particle & $a / \mathrm{sr}^{-1}$ & $b$ & $c$ & $d / \mathrm{sr}^{-1}$ \\
\hline$e^{-}$ & 4.27033 & -6.02527 & 0.29887 & -0.00103 \\
$e^{+}$ & 4.27725 & -6.02430 & 0.29856 & -0.00104 \\
$\gamma$ & 4.25716 & -6.02421 & 0.29926 & -0.00101 \\
\hline
\end{tabular}

Table B.5: Averaged parameters describing the angular distribution of emitted Cherenkov-light, section 3.5 . 


\begin{tabular}{|c|c|c|c|}
\hline particle & energy/ GeV & $a$ & $b$ \\
\hline \multirow[t]{13}{*}{$e^{-}$} & 1 & 1.96883 & 0.62794 \\
\hline & 3 & 2.68228 & 0.61705 \\
\hline & 7 & 3.30523 & 0.64303 \\
\hline & 10 & 3.61481 & 0.65247 \\
\hline & 30 & 4.16566 & 0.62248 \\
\hline & 70 & 4.77945 & 0.62823 \\
\hline & 100 & 4.98860 & 0.63416 \\
\hline & 300 & 5.61779 & 0.62033 \\
\hline & 700 & 6.10809 & 0.62129 \\
\hline & 1000 & 6.24439 & 0.61083 \\
\hline & 3000 & 7.03624 & 0.63287 \\
\hline & 7000 & 7.60499 & 0.64319 \\
\hline & 10000 & 7.86789 & 0.65099 \\
\hline \multirow[t]{13}{*}{$e^{+}$} & 1 & 1.93375 & 0.61535 \\
\hline & 3 & 2.74487 & 0.63234 \\
\hline & 7 & 3.27811 & 0.62541 \\
\hline & 10 & 3.55064 & 0.64072 \\
\hline & 30 & 4.22803 & 0.63283 \\
\hline & 70 & 4.77295 & 0.62917 \\
\hline & 100 & 4.88106 & 0.61512 \\
\hline & 300 & 5.55997 & 0.61605 \\
\hline & 700 & 6.05207 & 0.61644 \\
\hline & 1000 & 6.30800 & 0.62317 \\
\hline & 3000 & 7.01259 & 0.63265 \\
\hline & 7000 & 7.58980 & 0.64096 \\
\hline & 10000 & 7.89891 & 0.65069 \\
\hline \multirow[t]{13}{*}{$\gamma$} & 1 & 2.49299 & 0.60823 \\
\hline & 3 & 3.66575 & 0.71860 \\
\hline & 7 & 3.99721 & 0.66217 \\
\hline & 10 & 4.10746 & 0.64095 \\
\hline & 30 & 5.08856 & 0.68042 \\
\hline & 70 & 5.33660 & 0.63890 \\
\hline & 100 & 5.60790 & 0.64568 \\
\hline & 300 & 6.08445 & 0.62277 \\
\hline & 700 & 6.61645 & 0.63321 \\
\hline & 1000 & 6.78153 & 0.62575 \\
\hline & 3000 & 7.47467 & 0.64408 \\
\hline & 7000 & 7.96892 & 0.64825 \\
\hline & 10000 & 8.13041 & 0.65240 \\
\hline
\end{tabular}

Table B.7: Results of the fits of the longitudinal cascade development, section 3.3 


\begin{tabular}{|c|c|c|c|c|c|}
\hline particle & energy/ GeV & $a / \mathrm{sr}^{-1}$ & $b$ & $c$ & $d / \mathrm{sr}^{-1}$ \\
\hline \multirow[t]{12}{*}{$e^{-}$} & 3 & 4.21013 & -6.01199 & 0.30008 & -0.00099 \\
\hline & 7 & 4.21865 & -6.02752 & 0.30088 & -0.00096 \\
\hline & 10 & 4.28687 & -6.02147 & 0.29801 & -0.00108 \\
\hline & 30 & 4.29190 & -6.02932 & 0.29836 & -0.00103 \\
\hline & 70 & 4.29202 & -6.02913 & 0.29837 & -0.00104 \\
\hline & 100 & 4.24274 & -6.02275 & 0.29962 & -0.00098 \\
\hline & 300 & 4.28351 & -6.02684 & 0.29850 & -0.00104 \\
\hline & 700 & 4.28365 & -6.02672 & 0.29850 & -0.00104 \\
\hline & 1000 & 4.28351 & -6.02679 & 0.29849 & -0.00103 \\
\hline & 3000 & 4.28364 & -6.02685 & 0.29850 & -0.00104 \\
\hline & 7000 & 4.28360 & -6.02690 & 0.29850 & -0.00104 \\
\hline & 10000 & 4.28365 & -6.02689 & 0.29850 & -0.00103 \\
\hline \multirow[t]{12}{*}{$e^{+}$} & 3 & 4.38344 & -6.03618 & 0.29571 & -0.00113 \\
\hline & 7 & 4.19912 & -6.00949 & 0.30022 & -0.00095 \\
\hline & 10 & 4.24809 & -6.01802 & 0.29919 & -0.00105 \\
\hline & 30 & 4.24807 & -6.01820 & 0.29918 & -0.00104 \\
\hline & 70 & 4.28043 & -6.02557 & 0.29851 & -0.00103 \\
\hline & 100 & 4.28069 & -6.02577 & 0.29853 & -0.00104 \\
\hline & 300 & 4.28116 & -6.02627 & 0.29857 & -0.00105 \\
\hline & 700 & 4.28100 & -6.02623 & 0.29854 & -0.00103 \\
\hline & 1000 & 4.28106 & -6.02620 & 0.29854 & -0.00103 \\
\hline & 3000 & 4.28124 & -6.02647 & 0.29856 & -0.00104 \\
\hline & 7000 & 4.28125 & -6.02656 & 0.29856 & -0.00103 \\
\hline & 10000 & 4.28134 & -6.02658 & 0.29856 & -0.00103 \\
\hline \multirow[t]{12}{*}{$\gamma$} & 3 & 4.15186 & -6.02039 & 0.30274 & -0.00085 \\
\hline & 7 & 4.14478 & -6.00543 & 0.30202 & -0.00093 \\
\hline & 10 & 4.30725 & -6.02368 & 0.29745 & -0.00107 \\
\hline & 30 & 4.31395 & -6.03350 & 0.29786 & -0.00103 \\
\hline & 70 & 4.25518 & -6.02358 & 0.29922 & -0.00099 \\
\hline & 100 & 4.25584 & -6.02391 & 0.29928 & -0.00101 \\
\hline & 300 & 4.25586 & -6.02383 & 0.29926 & -0.00101 \\
\hline & 700 & 4.25605 & -6.02399 & 0.29926 & -0.00101 \\
\hline & 1000 & 4.28625 & -6.02812 & 0.29849 & -0.00103 \\
\hline & 3000 & 4.28632 & -6.02803 & 0.29849 & -0.00103 \\
\hline & 7000 & 4.28625 & -6.02805 & 0.29848 & -0.00103 \\
\hline & 10000 & 4.28624 & -6.02800 & 0.29848 & -0.00103 \\
\hline
\end{tabular}

Table B.9: Results of fits of the angular distribution of emitted Cherenkov-light, section 3.5 . 


\begin{tabular}{llccc}
\hline particle & energy/GeV & $\mathrm{b}$ & $\phi_{0} / \mathrm{rad}$ & $\sigma$ \\
\hline$e^{-}$ & 1 & 0.07064716 & 3.47699427 & 0.02103594 \\
& 10 & 0.02131804 & 3.49345733 & 0.00739830 \\
& 100 & 0.00643688 & 3.52309761 & 0.00234943 \\
1000 & 0.00202350 & 3.48731664 & 0.00074057 \\
& 10000 & 0.00067586 & 3.46473695 & 0.00023550 \\
\hline$e^{+}$ & 1 & 0.07112344 & 3.48404544 & 0.02099490 \\
& 10 & 0.02097273 & 3.51963176 & 0.00724243 \\
& 100 & 0.00663420 & 3.48056236 & 0.00232045 \\
& 1000 & 0.00213402 & 3.46339344 & 0.00073926 \\
& 10000 & 0.00065039 & 3.50744179 & 0.00023639 \\
\hline$\gamma$ & 1 & 0.06652718 & 3.55998809 & 0.02135593 \\
& 10 & 0.02166958 & 3.47688180 & 0.00730116 \\
& 100 & 0.00637762 & 3.52556673 & 0.00235480 \\
& 1000 & 0.00207235 & 3.48507985 & 0.00074559 \\
10000 & 0.00066903 & 3.47379137 & 0.00023858 \\
\hline
\end{tabular}

Table B.10: Results of fits to the shower asymmetry, Eq. 16 section 3.6 . The used bin size is $30^{\circ}$. The column $\sigma$ gives the average standard deviation of fluctuations in each bin.

\begin{tabular}{ll}
\hline particle & $p / \mathrm{rad}^{-2}$ \\
\hline$e^{-}$ & 0.07029 \\
$e^{+}$ & 0.07064 \\
$\gamma$ & 0.06668 \\
\hline
\end{tabular}

Table B.11: Results of the amplitude coefficient of the shower asymmetry, Eq. 17 section 3.6 . 


\begin{tabular}{llcccc}
\hline particle & energy/ GeV & $\mathrm{a}$ & $\mathrm{b}$ & $\mathrm{c}$ & $\mathrm{d}$ \\
\hline$e^{-}$ & 3 & 142.9460887 & -9.57395706 & 0.15172749 & -0.00266262 \\
& 7 & 85.84316237 & -9.05308106 & 0.16313946 & -0.00257812 \\
10 & 77.91266880 & -8.91314499 & 0.16406813 & -0.00284706 \\
30 & 46.82551912 & -8.41395678 & 0.17847633 & -0.00255785 \\
70 & 38.02891569 & -8.19061093 & 0.18434614 & -0.00257831 \\
100 & 34.69834063 & -8.10546380 & 0.18758322 & -0.00247138 \\
300 & 28.97457908 & -7.91471330 & 0.19335737 & -0.00247902 \\
700 & 25.83417400 & -7.79936480 & 0.19747241 & -0.00242657 \\
1000 & 24.40584315 & -7.74365060 & 0.19963483 & -0.00238802 \\
3000 & 23.00296659 & -7.68029984 & 0.20171506 & -0.00238785 \\
7000 & 21.74138458 & -7.62373034 & 0.20390902 & -0.00236181 \\
10000 & 21.46673564 & -7.61084235 & 0.20439010 & -0.00235443 \\
\hline$e^{+}$ & 3 & 137.9670566 & -9.54748394 & 0.15276498 & -0.00256487 \\
7 & 84.48637367 & -9.00950972 & 0.16249928 & -0.00266916 \\
10 & 71.63054893 & -8.84968017 & 0.16696350 & -0.00265524 \\
& 43.90262329 & -8.34648184 & 0.18034774 & -0.00257627 \\
& 39.84186034 & -8.23419693 & 0.18270563 & -0.00258497 \\
70 & 34.87615415 & -8.10856986 & 0.18734870 & -0.00249897 \\
100 & 29.07673188 & -7.91861470 & 0.19324693 & -0.00248046 \\
300 & 25.89164868 & -7.80387042 & 0.19747634 & -0.00240558 \\
700 & 25.19834484 & -7.77379969 & 0.19835006 & -0.00241144 \\
1000 & 23.01752709 & -7.68236819 & 0.20174971 & -0.00237442 \\
3000 & 21.65536829 & -7.62068764 & 0.20410342 & -0.00235278 \\
7000 & 21.33114755 & -7.60423566 & 0.20462732 & -0.00235435 \\
\hline 10000 & 69.70469246 & -8.90640987 & 0.17075128 & -0.00211751 \\
& 3 & 58.54718763 & -8.65787517 & 0.17279750 & -0.00249754 \\
7 & 60.52821188 & -8.64481073 & 0.17011384 & -0.00280772 \\
10 & 46.94065239 & -8.39738538 & 0.17763706 & -0.00264893 \\
30 & 33.61049111 & -8.06982820 & 0.18848567 & -0.00247429 \\
70 & 31.15779106 & -7.99239921 & 0.19102320 & -0.00247773 \\
100 & 26.69846316 & -7.83299913 & 0.19630007 & -0.00244224 \\
300 & 24.57774841 & -7.74984999 & 0.19933205 & -0.00239464 \\
700 & 23.43128734 & -7.70044416 & 0.20109158 & -0.00238882 \\
1000 & 22.16347238 & -7.64277275 & 0.20314619 & -0.00237112 \\
7000 & 20.95984696 & -7.58781685 & 0.20539395 & -0.00233737 \\
10000 & -71724506 & -7.57557901 & 0.20582216 & -0.00233654
\end{tabular}

Table B.13: Results of fits of Eq. (15) to the angular distribution of Cherenkov-light for the first third of the longitudinal cascade profile, section 3.7 . 


\begin{tabular}{|c|c|c|c|c|c|}
\hline particle & energy/GeV & $\mathrm{a}$ & $\mathrm{b}$ & $\mathrm{c}$ & d \\
\hline \multirow[t]{12}{*}{$e^{-}$} & 3 & 1.94993953 & -5.57898352 & 0.40890846 & 0.00136720 \\
\hline & 7 & 2.23159905 & -5.66592786 & 0.38702811 & 0.00096165 \\
\hline & 10 & 2.41790477 & -5.69781676 & 0.37321829 & 0.00074460 \\
\hline & 30 & 2.64370991 & -5.72687457 & 0.35830301 & 0.00037598 \\
\hline & 70 & 2.83551875 & -5.77447099 & 0.34890180 & 0.00017227 \\
\hline & 100 & 2.87505527 & -5.78793324 & 0.34732224 & 0.00017138 \\
\hline & 300 & 3.02408620 & -5.81360770 & 0.34020700 & 0.00000261 \\
\hline & 700 & 3.12021062 & -5.83135934 & 0.33600149 & -0.00008677 \\
\hline & 1000 & 3.14748514 & -5.83561542 & 0.33478931 & -0.00011422 \\
\hline & 3000 & 3.22395167 & -5.85249577 & 0.33188936 & -0.00018386 \\
\hline & 7000 & 3.28080118 & -5.86421471 & 0.32972990 & -0.00022818 \\
\hline & 10000 & 3.28101811 & -5.86419504 & 0.32973948 & -0.00022808 \\
\hline \multirow[t]{12}{*}{$e^{+}$} & 3 & 2.04418647 & -5.57393706 & 0.39850069 & 0.00104086 \\
\hline & 7 & 2.19550814 & -5.63219580 & 0.38766663 & 0.00100450 \\
\hline & 10 & 2.38067758 & -5.68162469 & 0.37507854 & 0.00073911 \\
\hline & 30 & 2.71866151 & -5.75661338 & 0.35520946 & 0.00036425 \\
\hline & 70 & 2.74306888 & -5.75739258 & 0.35363398 & 0.00031489 \\
\hline & 100 & 2.85095217 & -5.77142273 & 0.34768022 & 0.00014361 \\
\hline & 300 & 3.01522146 & -5.81294569 & 0.34066357 & 0.00001562 \\
\hline & 700 & 3.10293302 & -5.82808660 & 0.33673188 & -0.00006997 \\
\hline & 1000 & 3.14755994 & -5.83742082 & 0.33491531 & -0.00011240 \\
\hline & 3000 & 3.21892957 & -5.85126920 & 0.33206727 & -0.00017664 \\
\hline & 7000 & 3.26963124 & -5.86055633 & 0.33005267 & -0.00022419 \\
\hline & 10000 & 3.30250950 & -5.86852133 & 0.32892921 & -0.00024216 \\
\hline \multirow[t]{12}{*}{$\gamma$} & 3 & 2.31187235 & -5.66208473 & 0.37959321 & 0.00077227 \\
\hline & 7 & 2.41282189 & -5.68451332 & 0.37267906 & 0.00068690 \\
\hline & 10 & 2.51707194 & -5.69019396 & 0.36481000 & 0.00052426 \\
\hline & 30 & 2.75219082 & -5.76825072 & 0.35376221 & 0.00034959 \\
\hline & 70 & 2.86679434 & -5.77857129 & 0.34716252 & 0.00015492 \\
\hline & 100 & 2.98152659 & -5.80586065 & 0.34217497 & 0.00003287 \\
\hline & 300 & 3.06538269 & -5.81769690 & 0.33811151 & -0.00004781 \\
\hline & 700 & 3.16811393 & -5.84135741 & 0.33410858 & -0.00013899 \\
\hline & 1000 & 3.19153423 & -5.84578346 & 0.33314079 & -0.00015419 \\
\hline & 3000 & 3.26243729 & -5.86085963 & 0.33046056 & -0.00020943 \\
\hline & 7000 & 3.30585081 & -5.86779646 & 0.32870484 & -0.00025242 \\
\hline & 10000 & 3.30895722 & -5.87088305 & 0.32877273 & -0.00024303 \\
\hline
\end{tabular}

Table B.15: Results of fits of Eq. 15) to the angular distribution of Cherenkov-light for the middle third of the longitudinal cascade profile, section 3.7 


\begin{tabular}{|c|c|c|c|c|c|}
\hline particle & energy/GeV & $\mathrm{a}$ & $\mathrm{b}$ & $\mathrm{c}$ & d \\
\hline \multirow[t]{12}{*}{$e^{-}$} & 3 & 142.6044855 & -9.57205854 & 0.15179690 & -0.00265801 \\
\hline & 7 & 85.99022343 & -9.05411822 & 0.16307753 & -0.00257921 \\
\hline & 10 & 77.79962657 & -8.91179784 & 0.16410414 & -0.00284619 \\
\hline & 30 & 46.88367741 & -8.41476975 & 0.17842752 & -0.00255845 \\
\hline & 70 & 38.08621359 & -8.19192075 & 0.18429858 & -0.00257872 \\
\hline & 100 & 34.72957609 & -8.10617168 & 0.18754369 & -0.00247331 \\
\hline & 300 & 28.97315954 & -7.91468463 & 0.19335970 & -0.00247882 \\
\hline & 700 & 25.95546681 & -7.80308554 & 0.19724201 & -0.00243642 \\
\hline & 1000 & 24.48558230 & -7.74579464 & 0.19946929 & -0.00239809 \\
\hline & 3000 & 23.04520886 & -7.68159582 & 0.20162205 & -0.00239248 \\
\hline & 7000 & 21.71336645 & -7.62283497 & 0.20397007 & -0.00235902 \\
\hline & 10000 & 21.46127003 & -7.61065501 & 0.20440436 & -0.00235375 \\
\hline \multirow[t]{12}{*}{$e^{+}$} & 3 & 138.1008936 & -9.54847446 & 0.15274270 & -0.00256489 \\
\hline & 7 & 84.49824682 & -9.00981190 & 0.16249033 & -0.00266739 \\
\hline & 10 & 71.69254483 & -8.85031418 & 0.16693985 & -0.00265661 \\
\hline & 30 & 43.95573617 & -8.34754329 & 0.18030217 & -0.00257905 \\
\hline & 70 & 39.85957512 & -8.23454455 & 0.18268712 & -0.00258586 \\
\hline & 100 & 34.87521933 & -8.10854937 & 0.18735001 & -0.00249890 \\
\hline & 300 & 29.07085848 & -7.91850474 & 0.19325786 & -0.00247968 \\
\hline & 700 & 25.88875951 & -7.80377086 & 0.19748135 & -0.00240549 \\
\hline & 1000 & 25.19809801 & -7.77379079 & 0.19835066 & -0.00241142 \\
\hline & 3000 & 23.01750888 & -7.68236750 & 0.20174975 & -0.00237442 \\
\hline & 7000 & 21.65536329 & -7.62068747 & 0.20410343 & -0.00235278 \\
\hline & 10000 & 21.33114596 & -7.60423560 & 0.20462733 & -0.00235435 \\
\hline \multirow[t]{12}{*}{$\gamma$} & 3 & 69.85074756 & -8.90835590 & 0.17069329 & -0.00211976 \\
\hline & 7 & 58.28180667 & -8.65424155 & 0.17295947 & -0.00248847 \\
\hline & 10 & 60.49942091 & -8.64435137 & 0.17012934 & -0.00280746 \\
\hline & 30 & 47.11516858 & -8.40045586 & 0.17750367 & -0.00265504 \\
\hline & 70 & 33.55526488 & -8.06827181 & 0.18855456 & -0.00247353 \\
\hline & 100 & 31.16182031 & -7.99249181 & 0.19101555 & -0.00247788 \\
\hline & 300 & 26.71881146 & -7.83341114 & 0.19625992 & -0.00244509 \\
\hline & 700 & 24.56636625 & -7.74960945 & 0.19935804 & -0.00239229 \\
\hline & 1000 & 23.43436187 & -7.70054100 & 0.20108516 & -0.00238910 \\
\hline & 3000 & 22.16109501 & -7.64269786 & 0.20315149 & -0.00237087 \\
\hline & 7000 & 20.96330326 & -7.58792493 & 0.20538521 & -0.00233778 \\
\hline & 10000 & 20.71783561 & -7.57559703 & 0.20582042 & -0.00233665 \\
\hline
\end{tabular}

Table B.17: Results of fits of Eq. 15) to the angular distribution of Cherenkov-light for the last third of the longitudinal cascade profile, section 3.7 


\section{Acknowledgement}

We thank the IceCube group at the RWTH Aachen University for fruitful discussions. We thank Dmitry Chirkin and Spencer Klein for reading the manuscript and valuable suggestions. This work is supported by the German Ministry for Education and Research (BMBF).

\section{References}

[1] A. Achterberg, et al., First year performance of the IceCube neutrino telescope, Astroparticle Physics 26 (3) (2006) 155-173.

[2] I. Belolaptikov, et al., The Baikal underwater neutrino telescope: Design, performance, and first results, Astroparticle Physics 7 (3) (1997) 263-282.

[3] M. Ageron, et al., ANTARES: the first undersea neutrino telescope, Nuclear Instruments and Methods in Physics Research Section A: Accelerators, Spectrometers, Detectors and Associated EquipmentArXiv:1104.1607v2 [astroph.IM].

[4] L. Kuzmichev, On the velocity of light signals in deep underwater neutrino experiments, Nuclear Instruments and Methods in Physics Research Section A: Accelerators, Spectrometers, Detectors and Associated Equipment 482 (1) (2002) 304-306, arXiv:hep-ex/0005036v1.

[5] P. Price, K. Woschnagg, Role of group and phase velocity in high-energy neutrino observatories, Astroparticle Physics 15 (1) (2001) 97-100, arXiv:hepex/0008001v1.

[6] K. Nakamura, et al., Review of particle physics, Journal of Physics G: Nuclear and Particle Physics 37 (2010) 075021.

[7] I. Frank, I. Tamm, On cerenkov radiation, Compt. rend. acad. sci. URSS 14 (1937) 109.

[8] R. Abbasi, et al., Calibration and characterization of the IceCube photomultiplier tube, Nuclear Instruments and Methods in Physics Research Section A: Accelerators, Spectrometers, Detectors and Associated Equipment 618 (1) (2010) 139-152.

[9] W. Heitler, The quantum theory of radiation, Dover Pubns, 1954. 
[10] D. Groom, Atomic and nuclear properties of materials for more than 300 materials, Particle Data Group Website (2012).

URL http://pdg.1bl.gov/2012/AtomicNuclearProperties/

[11] C. Wiebusch, The detection of faint light in deep underwater neutrino telescopes, Ph.D. thesis, RWTH Aachen university, pITHA 95/37, http://web.physik.rwth-aachen.de/ wiebusch/Publications/Various/phd.pdf (1995).

[12] M. Kowalski, On the cherenkov light emission of hadronic and electromagnetic cascades, AMANDA internal report AMANDA-IR/20020803, DESY-Zeuthen (August, 12 2002).

[13] K. Han, Simulation of Cascades Using GEANT4 for IceCube, IceCube internal report icecube/201104001, Physics and Astronomy Department, Canterbury University, New Zealand (March, 92005 ).

[14] R. Mirani, Parametrisation of EM-showers in the ANTARES detectorvolume, Ph.D. thesis, University of Amsterdam, doctoral thesis in computational physics (2002).

[15] R. Brun, F. Bruyant, M. Maire, A. McPherson, P. Zanarini, GEANT3 Users guide, Tech. rep., CERN DD/EE/84-1 (1987).

[16] S. Agostinelli, et al., GEANT4 - a simulation toolkit, Nuclear Instruments and Methods in Physics Research Section A: Accelerators, Spectrometers, Detectors and Associated Equipment 506 (3) (2003) 250-303.

[17] S. Razzaque, S. Seunarine, D. Z. Besson, D. W. McKay, J. P. Ralston, D. Seckel, Coherent radio pulses from geant generated electromagnetic showers in ice, Physical Review D 65 (10) (2002) 103002, arXiv:astro$\mathrm{ph} / 0112505 \mathrm{v} 3$.

[18] L. Rädel, C. Wiebusch, Calculation of the Cherenkov light yield from low energetic secondary particles accompanying high-energy muons in ice and water with GEANT-4 simulations, Astroparticle Physics 38 (2012) 53-67, arXiv:1206.5530 [astro-ph.IM].

[19] D. Chirkin, private communication, University of Wisconsin - Madison, USA (2012).

[20] Geant-Collaboration, Geant4 users guide for application developers, Accessible from the GEANT4 web page, http://geant4.cern.ch/, version 9.4 (2010). 
[21] G. Grindhammer, S. Peters, The parameterized simulation of electromagnetic showers in homogeneous and sampling calorimeters, Arxiv preprint hep-ex/0001020.

[22] G. Collaboration, Geant4 - physics reference manual, Accessible from the GEANT4 web page, http://geant4.cern.ch/, version 9.5.0 (2011).

[23] S. Klein, Suppression of bremsstrahlung and pair production due to environmental factors, Reviews of Modern Physics 71 (5) (1999) 1501.

[24] A. Schalicke, V. Ivanchenko, M. Maire, L. Urban, Improved description of bremsstrahlung for high-energy electrons in geant4, in: Nuclear Science Symposium Conference Record, 2008. NSS'08. IEEE, IEEE, 2008, pp. 2788-2791. 